\title{
Antisense oligonucleotide therapy for neurodegenerative disease
}

\author{
Richard A. Smith, ${ }^{1,2,3}$ Timothy M. Miller, ${ }^{1,4}$ Koji Yamanaka, ${ }^{1}$ Brett P. Monia, ${ }^{5}$ Thomas P. Condon, ${ }^{5}$ \\ Gene Hung, ${ }^{5}$ Christian S. Lobsiger, ${ }^{1}$ Chris M. Ward, ${ }^{1}$ Melissa McAlonis-Downes, ${ }^{1}$ Hongbing Wei, ${ }^{5}$ \\ Ed V. Wancewicz, ${ }^{5}$ C. Frank Bennett, ${ }^{5}$ and Don W. Cleveland ${ }^{1,4}$
}

${ }^{1}$ Ludwig Institute for Cancer Research, University of California, San Diego, La Jolla, California, USA. ${ }^{2}$ The Scripps Research Institute, La Jolla, California, USA. ${ }^{3}$ Center for Neurologic Study, La Jolla, California, USA. ${ }^{4}$ Department of Neurosciences, University of California, San Diego, La Jolla, California, USA. 5Isis Pharmaceuticals, Carlsbad, California, USA.

\begin{abstract}
Neurotoxicity from accumulation of misfolded/mutant proteins is thought to drive pathogenesis in neurodegenerative diseases. Since decreasing levels of proteins responsible for such accumulations is likely to ameliorate disease, a therapeutic strategy has been developed to downregulate almost any gene in the CNS. Modified antisense oligonucleotides, continuously infused intraventricularly, have been demonstrated to distribute widely throughout the CNS of rodents and primates, including the regions affected in the major neurodegenerative diseases. Using this route of administration, we found that antisense oligonucleotides to superoxide dismutase 1 (SOD1), one of the most abundant brain proteins, reduced both SOD1 protein and mRNA levels throughout the brain and spinal cord. Treatment initiated near onset significantly slowed disease progression in a model of amyotrophic lateral sclerosis (ALS) caused by a mutation in SOD1. This suggests that direct delivery of antisense oligonucleotides could be an effective, dosage-regulatable means of treating neurodegenerative diseases, including ALS, where appropriate target proteins are known.
\end{abstract}

\section{Introduction}

Neurodegenerative diseases such as Alzheimer disease, Parkinson disease, Huntington disease, and amyotrophic lateral sclerosis (ALS) have been linked to aggregated, toxic proteins (1). One therapeutic approach for such diseases is to reduce the levels of the accumulated proteins. Accomplishing this task is a challenge due in part to the limited permeability of the blood-brain barrier. This difficulty can be surmounted by directly injecting a viral vector encoding a transcription-based siRNA into a specific brain (2-4) or spinal cord (5) region or by exploiting retrograde delivery to spinal motor neurons after viral injection into muscle $(3,5-8)$. Current viral vectors, however, provide no mechanism for altering dosage or discontinuance of therapy and face significant challenges for reaching widespread areas of the CNS.

Short, synthetic oligonucleotides (15-25 nucleotides) bind by Watson-Crick hybridization to target mRNA in a sequence-specific manner. The mRNA in such a heteroduplex is a substrate for catalytic, intranuclear degradation by endogenous RNase H (9). The main hurdle for using antisense oligonucleotides in the brain and spinal cord is effective delivery to these tissues. Cerebral ventricles are cavities within the brain that contain cerebrospinal fluid (CSF) that is produced by the choroid plexus (10). Once produced, CSF circulates from the ventricles to all regions of the CNS, yielding complete replacement approximately 3 times a day (10). Exploiting this and the enhanced tolerability and potency of second-generation oligonucleotides (9) and the fact that drug administration by

Nonstandard abbreviations used: ALS, amyotrophic lateral sclerosis; CSF, cerebrospinal fluid; SOD, superoxide dismutase.

Conflict of interest: B.P. Monia, G. Hung, E.V. Wancewicz, and C.F. Bennett are employees of Isis Pharmaceuticals Inc. R.A. Smith and D.W. Cleveland are consultants to Isis Pharmaceuticals Inc. R.A. Smith and D.W. Cleveland and their respective institutions could materially benefit if a therapeutic product for treatment of ALS results from this work.

Citation for this article: J. Clin. Invest. 116:2290-2296 (2006). doi:10.1172/JCI25424. direct pumping into the CSF is routinely done in humans $(11,12)$, we describe herein an effective, dosage-regulatable method of gene therapy capable of reducing expression of genes within the CNS regions involved in the major human neurodegenerative diseases.

\section{Results}

A 20-mer oligonucleotide ( $\left.\mathrm{SOD}^{1146192}\right)$ targeting superoxide dismutase 1 (SOD1) with phosphorothioate modifications throughout and 2'-O-(2-methoxy)ethyl substitutions on the sugars of the first and last 5 nucleotides to increase biological half-lives and binding affinity (refs. 13-15; see Methods) was continuously pumped into the right lateral ventricle of a normal rat via a catheter surgically implanted through the skull and connected to an osmotic pump imbedded subcutaneously. Brain and spinal cord tissues were collected after 14 days of infusion, and concentrations of accumulated oligonucleotide were directly measured (Figure 1A). Significant oligonucleotide concentrations $(3.5-7 \mu \mathrm{M})$ were achieved not only in the brain and brainstem but also in all levels of the spinal cord after delivery to rats (Figure 1A). Similar concentrations were achieved after infusion into a nonhuman primate (Figure 1B), including in the lumbar spinal cord more that $25 \mathrm{~cm}$ from the site of infusion. These micromolar tissue concentrations are on par with the 1-6 micromolar concentrations of commonly used CNS active drugs such as antiseizure medications (16).

Similar infusion performed for 14 days with a tracking oligonucleotide (Isis ${ }^{13920}$ ) that shares the same chemical structure and is recognized by the monoclonal antibody 2E1 (17) confirmed delivery to all levels of the spinal cord, with prominent uptake in the ventral horn (Figure 1, C-E) as well as robust cellular uptake by lumbar motor neurons and the non-neuronal cells surrounding them, especially in primates (Figure 1, F-I). In addition, uptake of oligonucleotide in primates was demonstrated in the brain parenchyma relevant to neurodegenerative diseases, including the hippocampus, substantia nigra, pons, and cerebellum (Figure 1, J-M). Thus antisense oligo- 

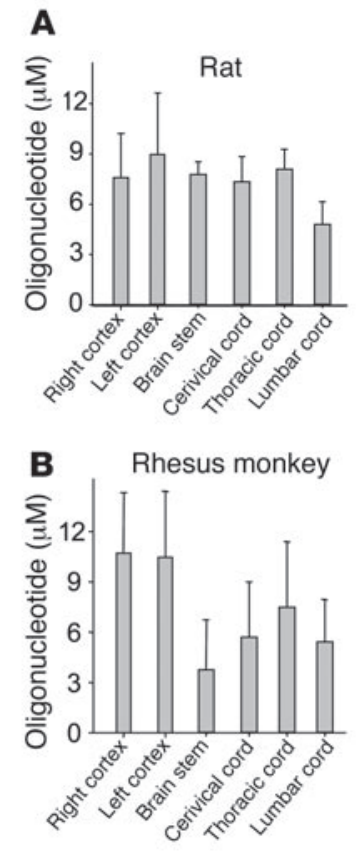

Rat lumbar ventral horn
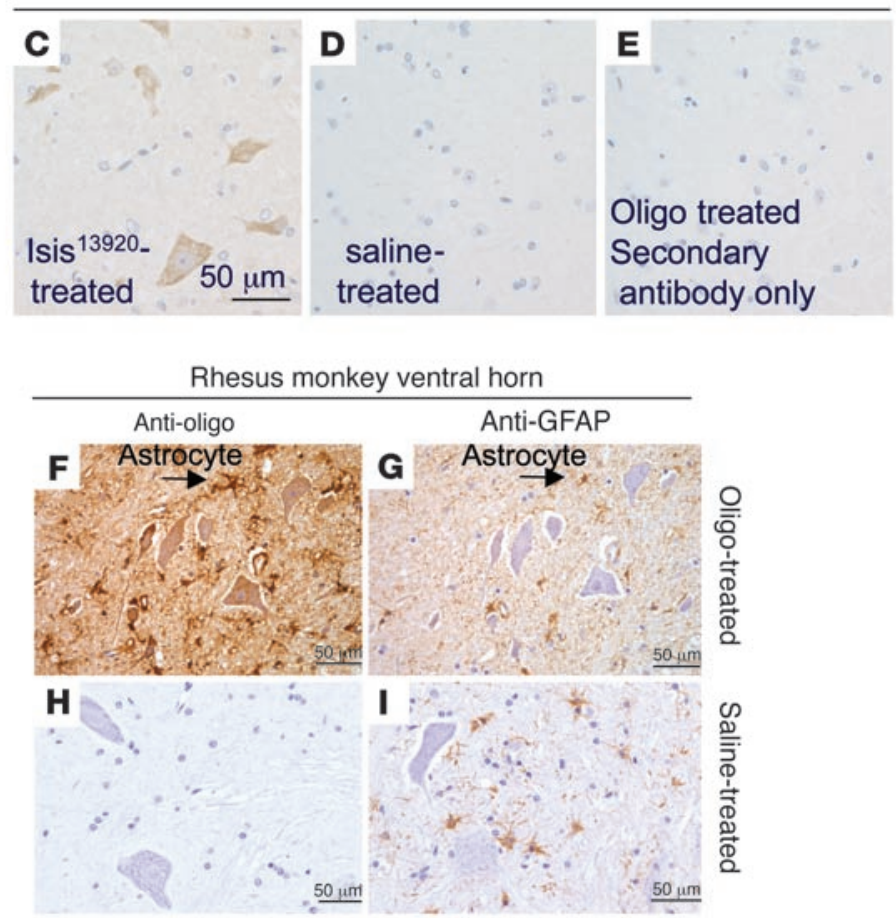

Rhesus monkey brain
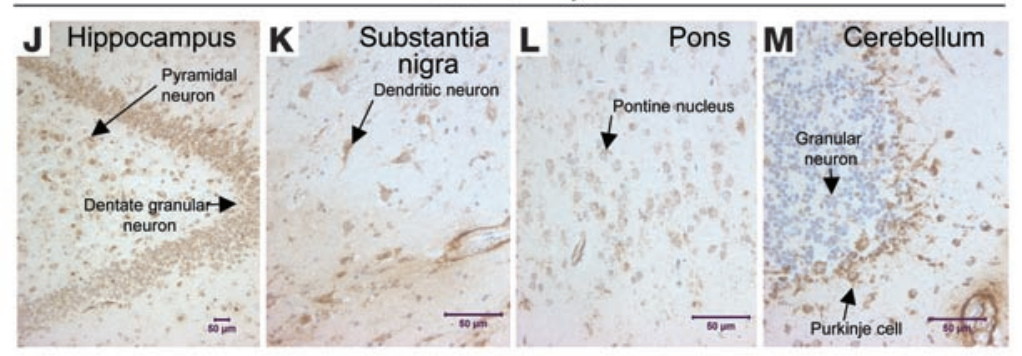

\section{Figure 1}

Distribution of antisense oligonucleotides after infusion into the right lateral ventricle in rats and Rhesus monkeys. ( $\mathbf{A}$ and $\mathbf{B}$ ) Antisense oligonucleotides were continuously infused for 2 weeks via infusion pump into the right lateral ventricle of normal rats at $100 \mu \mathrm{g} / \mathrm{d}(\mathbf{A})$ or Rhesus monkeys at $1 \mathrm{mg} / \mathrm{d}(\mathbf{B})$. Tissues were collected, and extracts were analyzed for oligonucleotide content by capillary gel electrophoresis. Mean \pm SD are shown ( $n=6$ [A]; 2 [B]). (C-M) A 24-mer modified oligonucleotide, Isis ${ }^{13920}$, was infused for 2 weeks into the right lateral ventricle at $100 \mu \mathrm{g} / \mathrm{d}$ in rats $(\mathbf{C}-\mathbf{E})$ or $1 \mathrm{mg} / \mathrm{d}$ in Rhesus monkeys (F-M). After perfusion, distribution of the oligonucleotide was determined by immunohistochemistry using a monoclonal antibody that recognizes the oligonucleotide $(\mathbf{C}-\mathbf{E}, \mathbf{F}, \mathbf{H}$, and $\mathbf{J}-\mathbf{M}$ ) or astrocytes (GFAP; $\mathbf{G}$ and $\mathbf{I})$. No oligonucleotide staining was seen in animals infused with saline only ( $\mathbf{D}$ and $\mathbf{H})$, nor in an animal infused with oligonucleotide but using secondary antibody only (E). Scale bars: $50 \mu \mathrm{m}$. cleotides, SOD ${ }^{\mathrm{r} / \mathrm{h} 146144}, \mathrm{SOD}^{\mathrm{r} / \mathrm{h} 146145}$, and SOD" 146192 (in which "r" and "h" denote complete complementarity to rat and human SOD1 mRNAs, respectively), reduced $S O D 1$ mRNA (Figure 2C) and protein (Figure 2D; kidney data not shown) in rat liver and kidney. The most effective one, SOD ${ }^{\text {r146192 }}$, reduced mRNA and SOD1 protein levels in liver to only $5 \%$ (Figure $2 \mathrm{~A}$ ) and 10\% (Figure $2 \mathrm{D}$ ) of normal, respectively, thus demonstrating decreased levels of the highly expressed SOD1 protein in tissues where adequate oligonucleotide concentrations are achieved. However, intraperitoneal administration did not affect brain or spinal cord SOD1 mRNA (Figure $2 \mathrm{C}$ ) or protein (data not shown), as expected from the minimal oligonucleotide concentrations in the CNS after peripheral administration $(20,21)$.

Continuous infusion of the SOD1 antisense oligonucleotide $\left(\mathrm{SOD}^{\mathrm{r146192}}\right)$, found to be most effective in cell culture, for 14 days into the right ventricle of normal rats effectively suppressed endogenous rat SOD1 mRNA, producing a dosedependent reduction to approximately $40 \%$ the normal level in cervical spinal cord (data not shown). An optimal dose of $100 \mu \mathrm{g} / \mathrm{d}$ was determined, above which no further reduction was seen. Infusion at this dosage for 28 days produced reduction in SOD1 mRNA levels to $25-40 \%$ of normal within the brain and at all spinal cord levels relative to infusion of saline only (Figure $3 \mathrm{~A})$. No diminution of accumulated SOD1 protein was seen after only 14 days of infusion, consistent with the long protein half-life of this very stably folded polypeptide (22). Infusion for 28 days, however, nucleotides not only distribute widely throughout the CNS of both rats and monkeys, but also penetrate deeply within tissues.

SOD 1 accumulates to approximately $1 \%$ of total brain protein (18), and mutations in the gene encoding it are responsible for $20 \%$ of familial ALS (19), a progressive neurodegenerative disease caused by preferential loss of neurons in the upper and lower motor pathways and for which current treatments are marginally beneficial at best. Oligonucleotides complementary to rat SOD1 mRNA that were effective in targeting it for degradation were identified initially by transfection into a cultured rat cell line (Figure 2A). Reduction of SOD1 mRNA levels in tissue culture was dose dependent (Figure 2B). Intraperitoneal injection into normal rats of 3 such oligonu- was accompanied by a $50 \%$ reduction in SOD1 protein levels in right frontal cortex and lumbar and cervical spinal cord (Figure 3, B-D). In each CNS compartment examined, suppression of SOD1 required complementarity of the oligonucleotide to SOD1 mRNA, as infusion of a scrambled oligonucleotide with identical composition and chemical modifications but altered sequence produced no change in either SOD1 mRNA or protein (Figure 3, A-D).

The feasibility of this gene downregulation strategy was further demonstrated by identifying antisense oligonucleotides that direct degradation of mRNAs encoding target proteins involved in Alzheimer disease, including presenilin 1, part of the $\gamma$-secretase complex that processes amyloid precursor protein to produce 

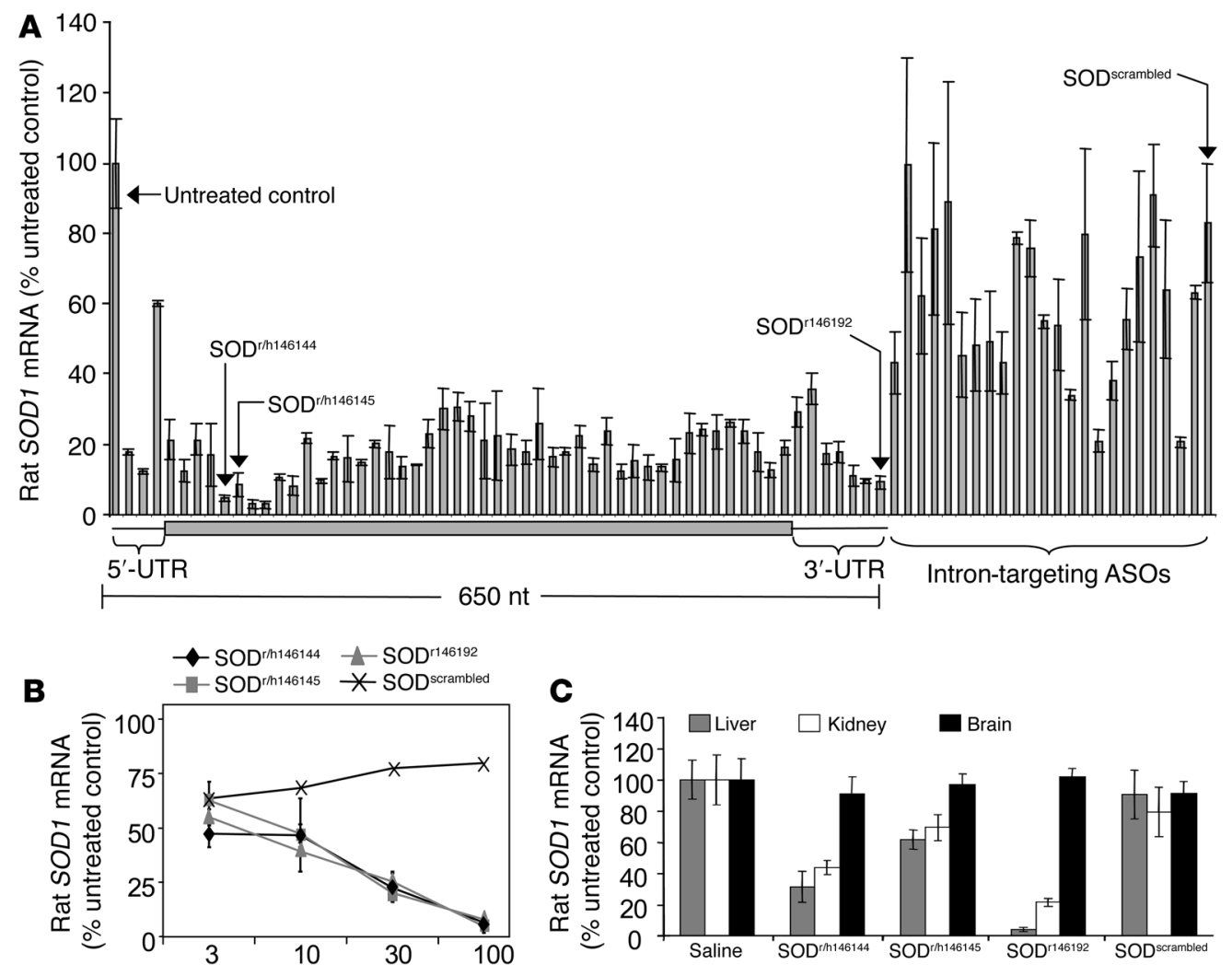

Oligonucleotide concentration (nM)
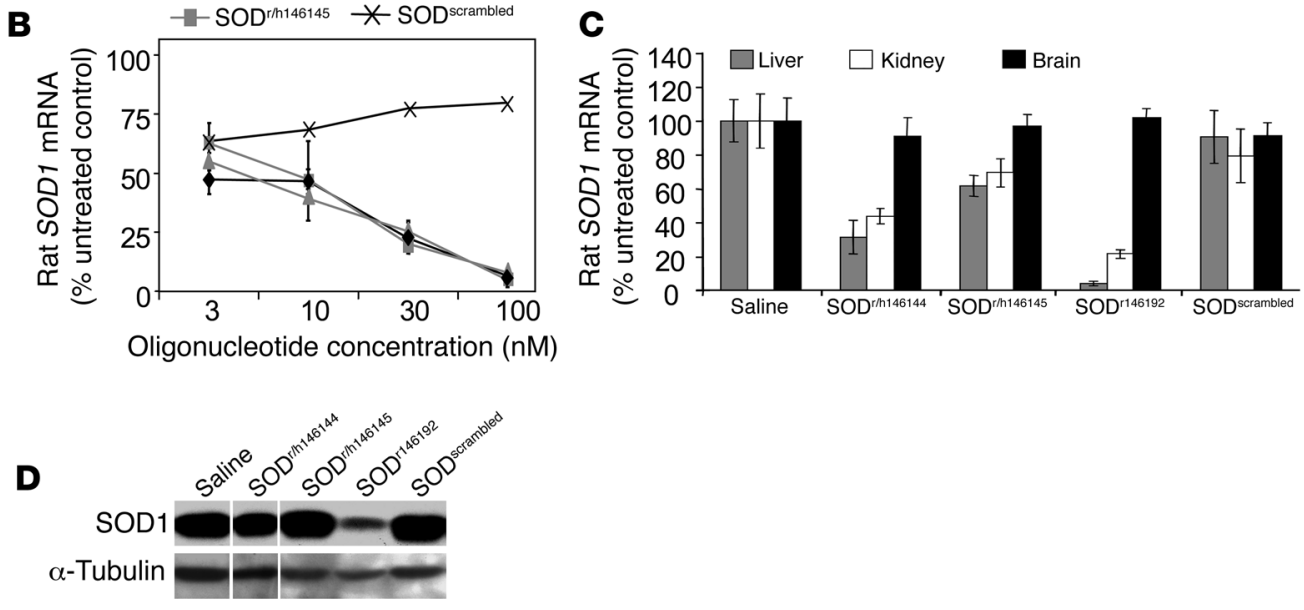

Figure 2

Identifying antisense oligonucleotides that reduce rat SOD1 in vitro and in vivo. (A) We synthesized seventyeight 24-mer, modified oligonucleotides complementary to rat SOD1 mRNA and transfected them at $150 \mathrm{nM}$ into primary rat A10 cells. RNA was prepared 24 hours after transfection, and SOD1 mRNA levels were measured by quantitative RT-PCR. Oligonucleotides are displayed relative to their positions on the 462-nucleotide SOD1 coding sequence. Mean \pm SD are shown $(n=4)$. UTR, untranslated region; ASOs, antisense oligonucleotides. (B) Oligonucleotides identified by the in vitro screen in A were evaluated in a similar transfection paradigm again using rat $\mathrm{A} 10$ cells and transfection of increasing concentrations of oligonucleotide to produce

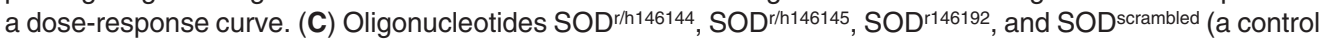
oligonucleotide) were injected $(37.5 \mathrm{mg} / \mathrm{kg}) 3$ times per week intraperitoneally into adult rats for 3 weeks, after which time mRNA levels were measured in the liver, kidney, and brain. Mean \pm SD are shown $(n=6)$. (D) SOD1 protein levels in liver extracts from animals treated with oligonucleotides SOD ${ }^{r / 146144}$, SOD $D^{r / h 146145}$, SOD ${ }^{\text {r146192 }}$, and SOD scrambled were measured by immunoblotting with an antibody to SOD1 (18). An immunoblot for tubulin was performed to verify protein loading.

the $A \beta$ peptide (23), and glycogen synthase kinase- $3 \beta$ (GSK-3 $\beta$ ), a kinase thought to be responsible for the aberrant phosphorylation of tau in intraneuronal tangles (24-26). Oligonucleotides effective in targeting presenilin 1 or GSK-3 3 mRNAs were identified by screening a series of oligonucleotides in cell culture for inhibition of their respective targets (data not shown). Intraventricular administration of the most effective of these for 14 days into normal mice substantially reduced the corresponding mRNAs in regions primarily affected in Alzheimer disease, including the right frontal/temporal cortex (Figure 3E).
Antisense oligonucleotides completely complementary to human SOD1 mRNA were synthesized, and the most effective at targeting human SOD1 mRNA degradation was determined by transfection into a human cell line. The best of these was tested for efficacy in a rat model that develops ALSlike progressive motor neuron disease from expression of a multicopy transgene encoding human ALS-linked mutant SOD $1{ }^{\mathrm{G} 93 \mathrm{~A}}$ to approximately $5 \%$ of total brain protein (27), that is, between 5 and 10 times the already abundant level found in endogenous wild-type rat SOD1. SOD ${ }^{\mathrm{r} / \mathrm{h3} 33611}$, the lead human oligonucleotide, suppressed $\mathrm{mRNA}$ and protein in vivo both after intraperitoneal administration (Figure 4A) and following intraventricular infusion for 1 month into SOD1 ${ }^{\mathrm{G} 93 \mathrm{~A}}$ transgenic rats (Figure 4, B-D). Directed degradation to approximately $20 \%$ the initial level of human SOD1 mRNA in the context of human RNA binding and processing components was demonstrated after introduction (by transfection) of oligonucleotide SOD ${ }^{\mathrm{r} / \mathrm{h} 333611}$ into fibroblasts from a symptomatic ALS patient carrying a mutant SOD1 $1^{\mathrm{A} 4 \mathrm{~V}}$ gene (Figure 5).

Oligonucleotide treatment to lower mutant human SOD1 ${ }^{\mathrm{G} 93 \mathrm{~A}}$ accumulation in the rat ALS model was initiated at 65 days of age $(\sim 30$ days prior to the age of disease onset; ref. 28) and rats were monitored for changes in weight, clinical signs of weakness, and survival. Infusion of saline only, $\mathrm{SOD}^{\mathrm{r} / \mathrm{h} 333611}$, and a scrambled oligonucleotide control produced indistinguishable disease onsets of $95 \pm 8(n=11), 95 \pm 10(n=12)$, and $97 \pm 13$ days $(n=8)$, respectively (Figure 6A). Early disease, as measured by loss of $10 \%$ of peak body weight, was similarly unaffected by treatment (saline, $111 \pm 6$ days; $\mathrm{SOD}^{\mathrm{r} / \mathrm{h} 333611}, 112 \pm 7$ days; scrambled oligonucleotide, $112 \pm 13$ days; Figure 6B). Remarkably, despite human SOD1 ${ }^{\mathrm{G} 93 \mathrm{~A}}$ accumulation to greater than $5 \%$ of total brain protein and the absence of slowing of early disease, infusion of SOD $\mathrm{D}^{\mathrm{r} / \mathrm{h} 333611}$ slowed disease progression, extending survival to $132 \pm 7$ days compared with $122 \pm 8$ days in saline-infused rats (Figure 6C), a 37\% extension of the typical 


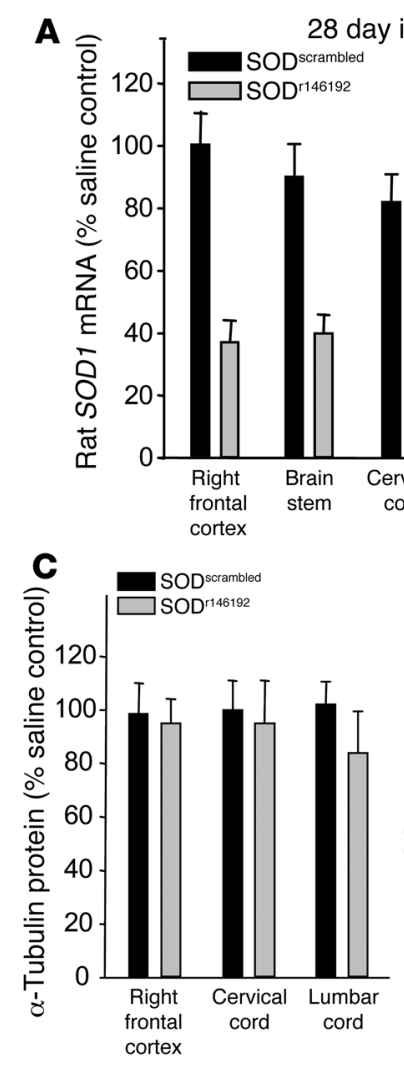

28 day infusion
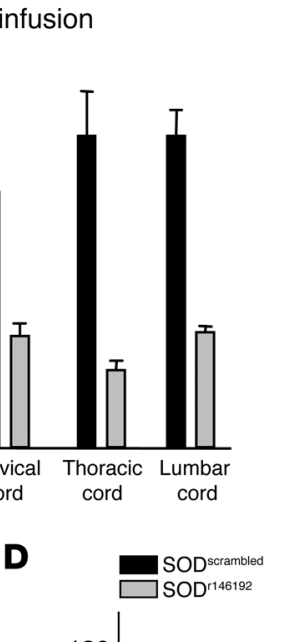

\section{E}

Right frontal/

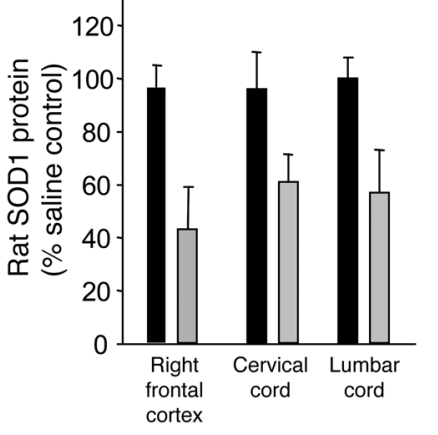

(⿸丆口⺕亅

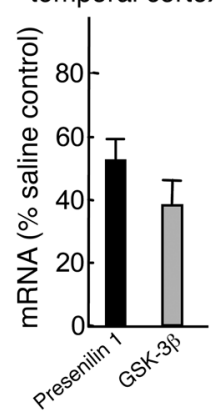

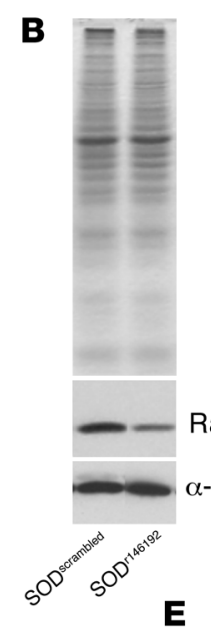

\section{Figure 3}

Antisense oligonucleotides reduce rat SOD1 in vivo. (A-D) Antisense SOD1 oligonucleotides SODr146192 or SODscrambled were infused for 28 days into the right lateral ventricle of normal rats at $100 \mu \mathrm{g} / \mathrm{d}$. (A) Endogenous SOD1 mRNA levels from brain and spinal cord regions were measured by quantitative real-time I RT-PCR. Mean \pm SD are shown $(n=6)$. (B) SOD1 and $\alpha$-tubulin protein levels were analyzed by immunoblotting following infusion. The Coomassie-stained gel at top demonstrates equal loading. (C and D) Protein levels for tubulin and SOD1 were quantified for right cortex, cervical cord, and lumbar cord after infusion as in B. Mean \pm SD are shown $(n=6)$. (E) Antisense oligonucleotides against presenilin 1 or GSK-3 $\beta$ were infused for 2 weeks into the right lateral ventricle of nontransgenic mice, and mRNA levels were measured by quantitative RT-PCR in the right frontal/temporal cortex $(n=6)$.

be adjusted noninvasively by remote signaling (11, 12). Surgical implantation and pump replenishing is routine, allowing infusion indefinitely $(11,12)$. Safety of locally and systemically administered oligonucleotides in patients is well established, with over 500 human subjects already exposed to oligonucleotides with the chemical backbone modifications used here (32). While siRNA to direct selective mRNA degradation using the multicomponent RNA-induced silencing complex (33) is an alternative to DNA oligonucleotides, the 27-day disease duration after onset. Survival after scrambled oligonucleotide infusion (121 \pm 9 days) was no different than saline.

\section{Discussion}

The strategy reported here provides an effective method for gene downregulation that may be applicable to multiple neurodegenerative diseases and incorporates 2 central advantages over current viral gene therapy approaches (29-31). Antisense oligonucleotide infusion can be regulated or stopped should there be any unforeseen side effects, a key consideration for human application. Wellestablished and sophisticated pump technology allows dosing to

\section{Figure 4}

Antisense oligonucleotides complementary to human SOD1 mRNA decrease SOD1 protein levels in SOD1G93A rat liver and spinal cord. (A) An oligonucleotide active against human SOD1 mRNA as well as a rat mRNA-specific oligonucleotide (SODr146192) was injected intraperitoneally 3 times per week $(37.5 \mathrm{mg} / \mathrm{kg}$ at a concentration of $3 \mathrm{M}$ ) into adult rats expressing a low copy number human SOD1G93A transgene (line L26L; ref. 27). After 3 weeks, liver extracts were prepared and analyzed by immunoblotting using an antibody that recognizes rat and human SOD1 with equal affinity (18). (B-D) Antisense oligonucleotides complementary to human SOD1 mRNA were infused into the right lateral ventricle of 65-day-old SOD1G93A rats at $100 \mu \mathrm{g} / \mathrm{d}$ for 28 days. (B) RNA was prepared from tissue extracts, and SOD1 RNA levels were measured by real-time RT-PCR. (C and D) Protein levels for SOD1 and $\alpha$-tubulin were analyzed in parallel extracts by immunoblotting with an antibody recognizing human and rat SOD1 with equal affinity (C) and were quantified for cervical cord (D). ${ }^{\star} P<0.05$ versus SOD ${ }^{\text {scrambled; }}$ Student's $t$ test. Mean \pm SD are shown ( $n=4$ [SODscrambled]; 8 [SOD//h333611]). more rapid turnover of siRNAs (even when modified to enhance stability) requires infusion of substantially higher dosages to target even relatively rare mRNAs in the CNS (34). Moreover, since siRNAs obligatorily bind to and can saturate components required for endogenous small RNA pathways, this can produce serious "off-target" consequences, including death, from dysregulation of endogenous small RNAs (35).
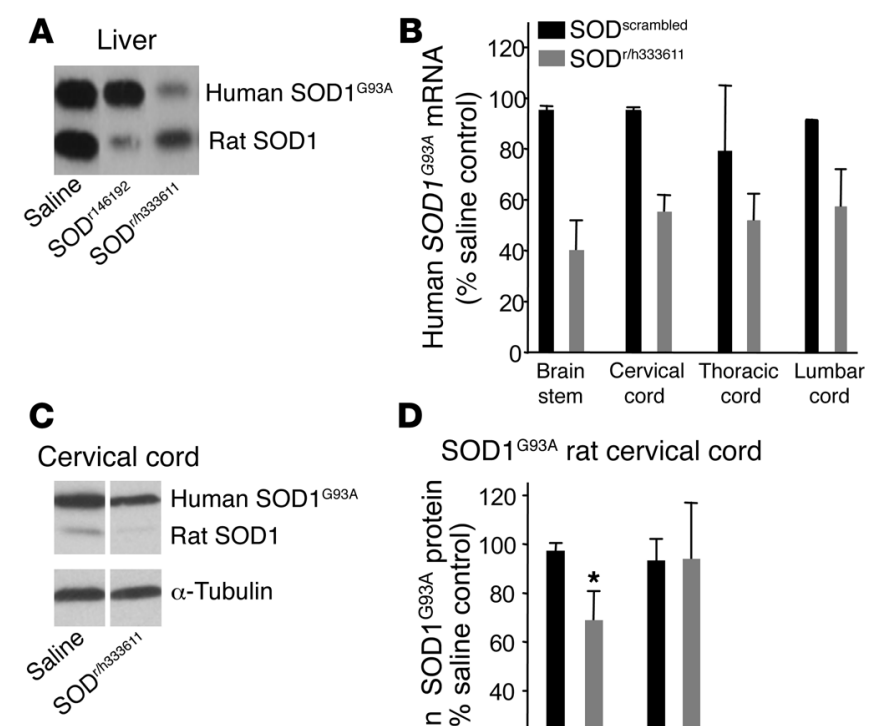

D

SOD1 $1^{\mathrm{G} 93 \mathrm{~A}}$ rat cervical cord

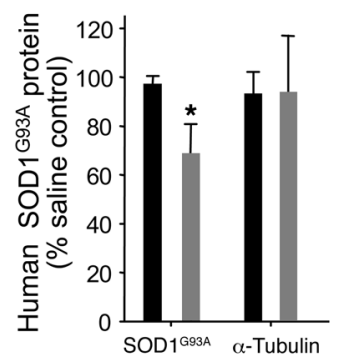




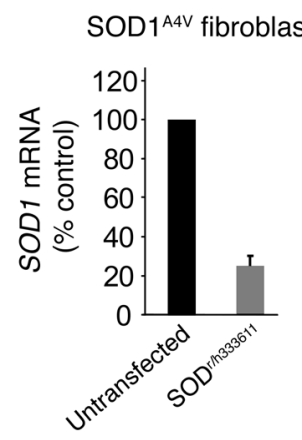

Figure 5

Antisense oligonucleotides decrease SOD1 ${ }^{A 4 V}$ in fibroblasts from an ALS patient. Fibroblasts from a patient meeting clinical criteria for ALS and heterozygous for the SOD1A4V mutation were transfected with $300 \mathrm{nM}$ of an oligonucleotide complementary to wild-type and mutant human SOD1 mRNA. Extracts were prepared after 48 hours and analyzed by quantitative RT-PCR for SOD1 mRNA levels $(n=4)$.

An additional benefit of the antisense oligonucleotide infusion method is widespread distribution and uptake in some or all of the non-neuronal cells within each CNS domain. For SOD1 mutantmediated ALS, toxicity to motor neurons is non-cell autonomous, with wild-type non-neuronal cells capable of delaying or eliminating degeneration and death of mutant-expressing motor neurons, and wild-type neurons acquiring damage from mutant-expressing neighbors (36). The slowing of disease after onset with oligonucleotide infusion contrasts with earlier gene therapy approaches using peripheral injection of an siRNA-encoding virus coupled with its delivery to motor neurons through retrograde transport $(6,7)$. These strategies failed to slow disease progression after onset, and in one instance disease progression was significantly accelerated (by 30\%; ref. 7) despite treatment initiated in juvenile (7-day-old) mice. These findings may be explained by failure to suppress mutant action in the non-neuronal cells.

Since diminished dismutase activity (37) or even complete absence of SOD1 (38) does not produce overt disease, compromise lifespan, or affect SOD1 mutant-mediated disease in mice (39), our results support infusion of antisense oligonucleotides as a treatment of familial ALS, especially for patients with the SOD $1^{\mathrm{A} 4 \mathrm{~V}}$ mutation, which accounts for approximately $50 \%$ of SOD1-mediated ALS in the United States and has an unusually rapid disease course after onset (40). The significantly lower normal levels of potential disease targets, including amyloid precursor protein (0.05\%; refs. 41,42$)$, presenilin 1 ( $0.0003 \%$; ref. 23$)$, tau (0.01- $0.1 \%$ of total brain protein; ref. 43 ), and $\alpha$-synuclein ( $0.5 \%$; ref. 44$)$, makes this approach even more attractive in these other diseases. This is especially so for targets that are nonessential genes whose absence does not compromise lifespan in mice, including amyloid precursor protein (45), the BACE protease, whose action is required to generate the $A \beta$ peptide in Alzheimer disease (46), and tau (47). Even for gene products for which loss of function has a phenotype (e.g., Huntington; refs. 48, 49; and presenilin 1; ref. 50), and which provide essential roles during early embryogenesis $(48,49)$, expression may not be necessary in adult neurons or their non-neuronal neighbors. Widespread oligonucleotide delivery in primates at levels sufficient to suppress even a very abundant mRNA (Figure 1) indicates that oligonucleotide therapy that effectively decreases the intended target can reach the relevant brain regions in human diseases including ALS, Alzheimer disease, frontal temporal dementia, Parkinson disease, and Huntington disease. If the formal safety studies underway in animals and the planned Phase I trial in human familial ALS patients demonstrate adequate safety (admittedly a substantial hurdle for any novel therapy), there are indeed many potential targets with a real possible impact on neurodegeneration.

\section{Methods}

Oligonucleotide preparation and synthesis and screening. For each target mRNA, 78 oligonucleotides corresponding to regions within the coding regions and $5^{\prime}$ and $3^{\prime}$ untranslated regions were synthesized and purified as described previously (51). Oligonucleotides were phosphorothioate-modified chimeric oligonucleotides composed of five 2'-O-(2-methoxy)ethyl modifications on both the $5^{\prime}$ and $3^{\prime}$ ends and 10 oligodeoxynucleotides in the center to support $\mathrm{RNase} \mathrm{H}$ activity (13). Initial in vitro screening was performed by transfection of oligonucleotide (at $150 \mathrm{nM}$ with $3.75 \mathrm{mg} / \mathrm{ml}$ Lipofectamine; Invitrogen) into a rat aortic cell line (A10 cells) for rat oligonucleotides and into a human lung cell line (A549 cells) for human oligonucleotides as described (13). Twenty-four hours after transfection, cells were harvested, and mRNA levels were measured by quantitative RT-PCR as described below. Oligonucleotide sequences were as follows, with underlined portions corresponding to 2 '-O(2-methoxy)ethyl modifications: SOD ${ }^{\mathrm{r} / \mathrm{h} 146145}$, $5^{\prime}$-GTCGCCCTTCAGCACGCACA-3'; SOD ${ }^{\mathrm{r} / \mathrm{h} 333611}$, 5'-CCGTCGCCCTTCAGCACGCA-3'; SOD ${ }^{\mathrm{r} / \mathrm{h} 141923}$ (SOD ${ }^{\text {scrambled }}$ control oligonucleotide), 5'-CCTTCCCTGAAGGTTCCTCC-3'; SOD $^{\text {1146192 }}, 5^{\prime}$-CTACAGTTTAGCAGGACAG-3'; GSK-3 $3,5^{\prime}$-TCTCATGATCTGGAGCTCTC-3'; and presenilin 1, 5'
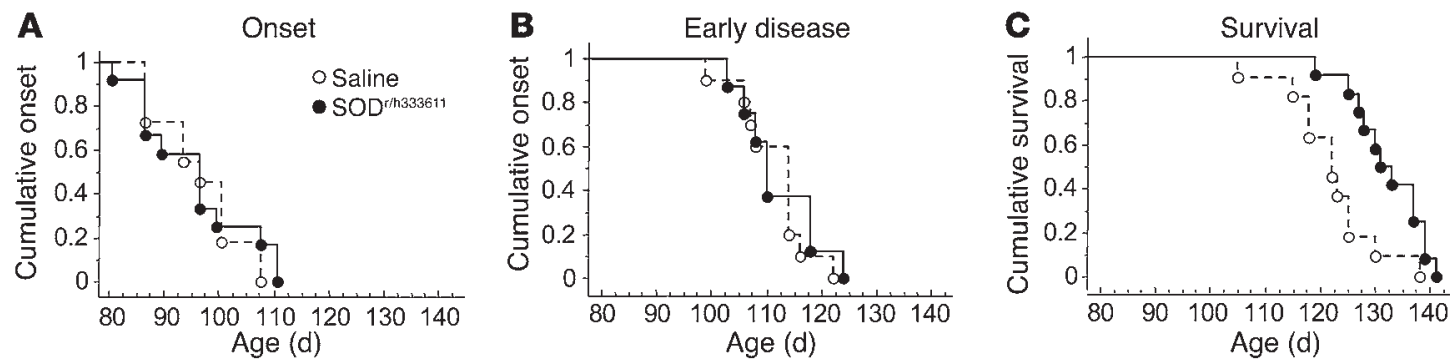

\section{Figure 6}

Infusion of oliogonucleotides complementary to human SOD1 mRNA extends survival in SOD1G93A rats. (A-C) Antisense oligonucleotides complementary to human SOD1 mRNA were infused into the right lateral ventricle of 65 -day-old SOD1G93A rats at $100 \mu \mathrm{g} / \mathrm{d}$ for 28 days $\left(n=11\right.$ [saline-infused]; 12 [SOD $\left.{ }^{r / 3333611}\right]$ ). Disease onset $(\mathbf{A})$ was defined as the peak animal weight, early disease (B) was defined as the point at which the animals had lost $10 \%$ of their peak weight, and survival (C) was defined as the inability of the animal to right itself within 30 seconds of being placed on its side. 
selected $\mathrm{SOD}^{\mathrm{r} / \mathrm{h} 141923}$ as a control oligonucleotide because it shares identical chemistry and length to the SOD1-targeted oligonucleotides, is not predicted to hybridize to any known human or rat genes, and was previously shown not to have detectable effects in tissue culture or in mouse models $(52,53)$.

Quantitative RT-PCR. mRNA levels were measured by a quantitative real-time RT-PCR method (54). Total RNA was isolated using an RNeasy Mini prep kit (QIAGEN) according to the manufacturer's protocol. We combined 5-10 ng total RNA with $100 \mathrm{nM}$ of each of the gene-specific dual-labeled probes and forward and reverse primers in a buffered solution consisting of $1 \times$ TaqMan Buffer A (Applied Biosystems), $5.5 \mathrm{mM}$ $\mathrm{MgCl}_{2}, 200 \mu \mathrm{M}$ concentrations of each dNTP (Amersham Biosciences), $2 \mathrm{U}$ RNase inhibitor, $0.625 \mathrm{U}$ AmpliTaq Gold, and $6.25 \mathrm{U}$ murine leukemia virus reverse transcriptase. Except for dNTP solutions, all reagents above were obtained from Applied Biosystems. Quantitative RT-PCR reactions were conducted and analyzed on an ABI Prism 7700 Sequence Detector (Applied Biosystems). Glyceraldehyde 3-phosphate dehydrogenase mRNA levels were used as an internal reference for normalization among samples. Primer probe set sequences for SOD1 were as follows: forward, $5^{\prime}$-TGCTGAAGGGCGACGG-3'; reverse, 5'-GTTCACCGCTTGCTTCTG-3'; probe, 5'-CCGGTGCAGGGCGTCATTCACTT-3'.

Quantitation of oligonucleotide concentrations. In order to determine the tissue concentration of oligonucleotides, approximately $100 \mathrm{mg}$ tissue were homogenized (Bio Savant, Bio $101 \mathrm{Inc}$.), phenol-chloroform was extracted, and solid phase was extracted using a phenyl-bonded SPE column (International Sorbent Technology) (55). Extracted samples were analyzed by capillary gel electrophoresis (CGE) using a Beckman P/ACE MDQ Model 5010 Capillary Electrophoresis instrument (Beckman Coulter) with UV detection at $260 \mathrm{~nm}$ (56). Quantitation of oligonucleotide was performed using standard curves of electropherogram peak area ratios (drug/internal standard) versus known concentrations over the anticipated concentration range or by the extinction coefficient method (56). Calibration curve and quality control samples were run with each unknown sample analysis. No oligonucleotide was detected by CGE in the saline-treated control samples. The limit of quantitation for the assay was estimated to be $0.35 \mu \mathrm{g} / \mathrm{g}(0.05 \mu \mathrm{M})$ oligonucleotide in tissue.

Surgical placement of osmotic pumps and harvesting tissues for analysis. SpragueDawley rats (65-75 days old) were anesthetized with intramuscular administration of ketamine, acepromazine, and xylazine. Subsequently, after sterilizing the surgical site with betadyne, a midline incision was made over the skull, and a subcutaneous pocket was created over the back. An osmotic pump (ALZET Osmotic Pumps) that had been filled with oligonucleotide and incubated overnight in saline at $37^{\circ} \mathrm{C}$ was implanted in the pocket. To deliver $100 \mu \mathrm{g} / \mathrm{d}$, oligonucleotides were diluted to $16.7 \mathrm{mg} / \mathrm{ml}$ in preservative-free, sterile $0.9 \%$ sodium chloride. The animal was placed in a stereotactic frame at which time a small burr hole was made through the skull immediately above the right lateral ventricle. A cannula, connected to the pump via a plastic catheter, was then placed in the ventricle, and the incision was manually closed with sutures. Depending on the experiment, oligonucleotide was infused for either 14 or 28 days, after which animals were anesthetized with isoflurane and sacrificed. Brain and spinal cord were rapidly removed (within approximately 5 minutes). RNA was immediately processed as described above. Tissues for subsequent analysis were placed in liquid nitrogen and stored at $-80^{\circ} \mathrm{C}$. The SOD1 ${ }^{\mathrm{G} 93 \mathrm{~A}}$ rat line $\mathrm{L} 26 \mathrm{~L}$ (27) (lower expressing) was used for initial evaluation of mRNA and protein levels. All animal protocols were approved by the UCSD Institutional Animal Care and Use Committee and met ethical standards for animal experimentation.

Assessment of onset and survival. For survival experiments, death was defined by paralysis so severe that the animal was unable to right itself within $30 \mathrm{sec}-$ onds when placed on its side, a common endpoint that is objective and minimizes distress for the experimental animals. Animals were weighed weekly. Onset was defined retrospectively by analyzing the first sign of weight loss. We defined $10 \%$ weight loss as the point when the animals lost $10 \%$ of their peak body weight. Nontransgenic control animals did not lose weight. For survival experiments, the SOD1 ${ }^{\mathrm{G} 93 \mathrm{~A}} \mathrm{~L} 26 \mathrm{H}$ (higher expressing) rat line was used.

Immunoblotting. Tissues for immunoblotting were thawed on ice and then homogenized in lysis buffer (1\% Triton X-100; 50 mM Tris-HCl, $\mathrm{pH} 7.5$; $150 \mathrm{mM} \mathrm{NaCl}$; and protease inhibitor mixture containing $1 \mu \mathrm{g} / \mathrm{ml}$ aprotinin, $1 \mu \mathrm{g} / \mathrm{ml}$ leupeptin, and $1 \mathrm{mM}$ PMSF). After centrifugation, the clarified supernatant was used for subsequent analysis. Lysates were resolved by $12 \%$ SDS/PAGE and transferred to nitrocellulose membranes (Schleicher $\&$ Schuell). Membranes were incubated with SOD1 antibody (18) or $\alpha$-tubulin antibody (Sigma-Aldrich) diluted 1:2,000 or 1:3,000, respectively, in 5\% nonfat skim milk/0.1\% Tween 20 in PBS, and were then incubated with Horseradish Peroxidase-conjugated Anti-Rabbit or Anti-Mouse IgG (Amersham Biosciences) and visualized with ECL (Amersham Biosciences). Immunoblots were quantified using Image J (a public domain Java image processing program inspired by NIH Image; http://rsb.info.nih.gov/ij/). Anti-GFAP antibodies were purchased from Abcam.

Infusion of oligonucleotides into Rhesus monkeys. Under anesthesia, catheters were surgically implanted in the left lateral ventricles of 5 Rhesus monkeys approximately 3 years of age, after which the animals recovered for 5 days. Catheters terminated in a subcutaneous titanium access port that was connected to an external pump housed within a jacket on the animal. Isis ${ }^{13920}$ (tracking oligonucleotide) was continuously infused into the left lateral ventricle of 2 animals at a concentration of $1.7 \mathrm{mg} / \mathrm{ml}$ in saline, for a total dose of $1 \mathrm{mg} / \mathrm{d}$. Control animals $(n=3)$ were infused with saline at a similar rate. No adverse behavioral effects were noted. After 14 days, animals were euthanized, and tissues were collected.

Statistics. Data were analyzed using 2-tailed Student's $t$ test. $P$ values less than 0.05 were considered significant.

\section{Acknowledgments}

This work has been supported by the Skaggs Clinical Scholars program at Scripps Clinic and Research Foundation (to R.A. Smith), a Lou Gehrig Challenge grant from the ALS Association (to D.W. Cleveland), NIH grant NS27036 (to D.W. Cleveland), NIH K12 grant (to T.M. Miller), and a Postdoctoral Fellowship from Uehara Memorial Foundation and Career Development Grant from the Muscular Dystrophy Association (to K. Yamanaka). D.W. Cleveland receives salary support from the Ludwig Institute for Cancer Research. The Center for Neurologic Study has been generously supported by the Thagard Foundation; the William Stephans Trust; Margaret McKenzie; James Schoensiegel; Daryl O. Smith; Basil Witt; and the Bobby Carter, Charles Donnelley, Rick Faber, Murry Sandler, and Jim Watkins ALS funds. Special thanks is accorded to Susan Donnelley, Richard Stephan, and Greg Thagard for advancing the cause of ALS and kindred disorders.

Received for publication April 21, 2005, and accepted in revised form June 6, 2006.

Address correspondence to: Timothy M. Miller, Neurosciences Department, University of California, San Diego, 9500 Gilman Drive, MC 0670, La Jolla, California 92093, USA. Phone: (858) 5347899; Fax: (858) 534-7659; E-mail: timiller@ucsd.edu. Or to: Richard A. Smith, Center for Neurologic Study, 9850 Genesee Avenue, Suite 320, La Jolla, California 92037, USA. Phone: (858) 455-5463; Fax: (858) 455-1713; E-mail: r8smith@ucsd.edu.

Richard A. Smith and Timothy M. Miller contributed equally to this work. 
1. Bossy-Wetzel, E., Schwarzenbacher, R., and Lipton, S.A. 2004. Molecular pathways to neurodegeneration. Nat. Med. 10(Suppl.):S2-S9.

2. Xia, H., Mao, Q., Paulson, H.L., and Davidson, B.L. 2002. siRNA-mediated gene silencing in vitro and in vivo. Nat. Biotechnol. 20:1006-1010.

3. Xia, H., et al. 2004. RNAi suppresses polyglutamine-induced neurodegeneration in a model of spinocerebellar ataxia. Nat. Med. 10:816-820.

4. Singer, O., et al. 2005. Targeting BACE1 with siRNAs ameliorates Alzheimer disease neuropathology in a transgenic model. Nat Neurosci. 8:1343-1349.

5. Raoul, C., et al. 2005. Lentiviral-mediated silencing of SOD1 through RNA interference retards disease onset and progression in a mouse model of ALS. Nat. Med. 11:423-428.

6. Miller, T.M., et al. 2005. Virus-delivered small RNA silencing sustains strength in amyotrophic lateral sclerosis. Ann. Neurol. 57:773-776.

7. Ralph, G.S., et al. 2005. Silencing mutant SOD1 using RNAi protects against neurodegeneration and extends survival in an ALS model. Nat. Med. 11:429-433.

8. Harper, S.Q., et al. 2005. RNA interference improves motor and neuropathological abnormalities in a Huntington's disease mouse model. Proc. Natl. Acad. Sci. U. S. A. 102:5820-5825.

9. Crooke, S.T. 2004. Progress in antisense technology. Annu. Rev. Med. 55:61-95.

10. Fishman, R.A. 1992. Cerebrospinal fluid in diseases of the nervous system. Saunders. Philadelphia, Pennsylvania, USA. $431 \mathrm{pP}$

11. Dash, A.K., and Cudworth, G.C., 2nd. 1998. Therapeutic applications of implantable drug delivery systems. J. Pharmacol. Toxicol. Methods. 40:1-12.

12. North, R.B., and Levy, R.M. 1997. Neurosurgical management of pain. Springer. New York, New York, USA. 360 pp.

13. McKay, R.A., et al. 1999. Characterization of a potent and specific class of antisense oligonucleotide inhibitor of human protein kinase C-alpha expression. J. Biol. Chem. 274:1715-1722.

14. Zhang, H., et al. 2000. Reduction of liver Fas expression by an antisense oligonucleotide protects mice from fulminant hepatitis. Nat. Biotechnol. 18:862-867.

15. Cook, P.D. 1998. Second generation antisense oligonucleotides: 2'-modifications. Annu. Rep. Med. Chem. 33:313-325.

16. Goodman, L.S., Hardman, J.G., Limbird, L.E., and Gilman, A.G. 2001. Goodman \& Gilman's the pharmacological basis of therapeutics. McGrawHill. New York, New York, USA. 2148 pp.

17. Butler, M., Stecker, K., and Bennett, C.F. 1997. Cellular distribution of phosphorothioate oligodeoxynucleotides in normal rodent tissues. Lab. Invest. 77:379-388.

18. Pardo, C.A., et al. 1995. Superoxide dismutase is an abundant component in cell bodies, dendrites, and axons of motor neurons and in a subset of other neurons. Proc. Natl. Acad. Sci. U. S. A. 92:954-958.

19. Rosen, D.R., et al. 1993. Mutations in Cu/Zn superoxide dismutase gene are associated with familial amyotrophic lateral sclerosis. Nature. 362:59-62.

20. Broaddus, W.C., Prabhu, S.S., Wu-Pong, S., Gillies, G.T., and Fillmore, H. 2000. Strategies for the design and delivery of antisense oligonucleotides in central nervous system. Methods Enzymol. 314:121-135.
21. Cossum, P.A., et al. 1993. Disposition of the 14Clabeled phosphorothioate oligonucleotide ISIS 2105 after intravenous administration to rats. J. Pharmacol. Exp. Ther. 267:1181-1190.

22. Borchelt, D.R., et al. 1994. Superoxide dismutase 1 with mutations linked to familial amyotrophic lateral sclerosis possesses significant activity. Proc. Natl. Acad. Sci. U. S. A. 91:8292-8296.

23. Thinakaran, G., et al. 1996. Endoproteolysis of presenilin 1 and accumulation of processed derivatives in vivo. Neuron. 117:181-190.

24. Lucas, J.J., et al. 2001. Decreased nuclear betacatenin, tau hyperphosphorylation and neurodegeneration in GSK-3beta conditional transgenic mice. $E M B O J .20: 27-39$

25. Noble, W., et al. 2005. Inhibition of glycogen synthase kinase- 3 by lithium correlates with reduced tauopathy and degeneration in vivo. Proc. Natl. Acad. Sci. U. S. A. 102:6990-6995

26. Phiel, C.J., Wilson, C.A., Lee, V.M., and Klein, P.S. 2003. GSK-3alpha regulates production of Alzheimer's disease amyloid-beta peptides. Nature. 423:435-439.

27. Howland, D.S., et al. 2002. Focal loss of the glutamate transporter EAAT2 in a transgenic rat model of SOD1 mutant-mediated amyotrophic lateral sclerosis (ALS). Proc. Natl. Acad. Sci. U. S. A. 99:1604-1609.

28. Liu, J., Shinobu, L.A., Ward, C.M., Young, D., and Cleveland, D.W. 2005. Elevation of the Hsp70 chaperone does not effect toxicity in mouse models of familial amyotrophic lateral sclerosis. J. Neurochem. 93:875-882.

29. Azzouz, M., et al. 2004. VEGF delivery with retrogradely transported lentivector prolongs survival in a mouse ALS model. Nature. 429:413-417.

30. Kaspar, B.K., Llado, J., Sherkat, N., Rothstein, J.D., and Gage, F.H. 2003. Retrograde viral delivery of IGF-1 prolongs survival in a mouse ALS model. Science. 301:839-842.

31. Dykxhoorn, D.M., Novina, C.D., and Sharp, P.A. 2003. Killing the messenger: short RNAs that silence gene expression. Nat. Rev. Mol. Cell Biol. 4:457-467.

32. Crooke, S.T. 2004. Antisense strategies. Curr. Mol. Med. 4:465-487.

33. Zamore, P.D., Tuschl, T., Sharp, P.A., and Bartel, D.P. 2000. RNAi: double-stranded RNA directs the ATP-dependent cleavage of mRNA at 21 to 23 nucleotide intervals. Cell. 101:25-33.

34. Dorn, G., et al. 2004. siRNA relieves chronic neuropathic pain. Nucleic Acids Res. 32:1-6.

35. Grimm, D., et al. 2006. Fatality in mice due to oversaturation of cellular microRNA/short hairpin RNA pathways. Nature. 441:537-541

36. Clement, A.M., et al. 2003. Wild-type nonneurona cells extend survival of SOD1 mutant motor neurons in ALS mice. Science. 302:113-117.

37. Subramaniam, J.R., et al. 2002. Mutant SOD1 causes motor neuron disease independent of copper chaperone-mediated copper loading. Nat. New rosci. 5:301-307.

38. Reaume, A.G., et al. 1996. Motor neurons in Cu/Zn superoxide dismutase-deficient mice develop normally but exhibit enhanced cell death after axonal injury. Nat. Genet. 13:43-47.

39. Bruijn, L.I., et al. 1998. Aggregation and motor neuron toxicity of an ALS-linked SOD1 mutant independent from wild-type SOD1. Science.
281:1851-1854

40. Cudkowicz, M.E., et al. 1997. Epidemiology of mutations in superoxide dismutase in amyotrophic lateral sclerosis. Ann. Neurol. 41:210-221.

41. Price, D.L., and Sisodia, S.S. 1998. Mutant genes in familial Alzheimer's disease and transgenic models. Annu. Rev. Neurosci. 21:479-505.

42. Potempska, A., Styles, J., Mehta, P., Kim, K.S., and Miller, D.L. 1991. Purification and tissue level of the beta-amyloid peptide precursor of rat brain. J. Biol. Chem. 266:8464-8469.

43. Cleveland, D.W., Hwo, S.Y., and Kirschner, M.W. 1977. Purification of tau, a microtubule-associated protein that induces assembly of microtubules from purified tubulin. J. Mol. Biol. 116:207-225.

44. Iwai, A., et al. 1995. The precursor protein of non-A beta component of Alzheimer's disease amyloid is a presynaptic protein of the central nervous system. Neuron. 14:467-475

45. Zheng, H., et al. 1995. beta-Amyloid precursor protein-deficient mice show reactive gliosis and decreased locomotor activity. Cell. 81:525-531.

46. Cai, H., et al. 2001. BACE1 is the major beta-secretase for generation of Abeta peptides by neurons. Nat. Neurosci. 4:233-234.

47. Harada, A., et al. 1994. Altered microtubule organization in small-calibre axons of mice lacking tau protein. Nature. 369:488-491.

48. Zeitlin, S., Liu, J.P., Chapman, D.L., Papaioannou, V.E., and Efstratiadis, A. 1995. Increased apoptosis and early embryonic lethality in mice nullizygous for the Huntington's disease gene homologue. Nat. Genet. 11:155-163.

49. Nasir, J., et al. 1995. Targeted disruption of the Huntington's disease gene results in embryonic lethality and behavioral and morphological changes in heterozygotes. Cell. 81:811-823.

50. Taylor, J.P., Hardy, J., and Fischbeck, K.H. 2002. Toxic proteins in neurodegenerative disease. Science. 296:1991-1995.

51. Cheruvallath, Z.S., Kumar, R.K., Rentel, C., Cole, D.L., and Ravikumar, V.T. 2003. Solid phase synthesis of phosphorothioate oligonucleotides utilizing diethyldithiocarbonate disulfide (DDD) as an efficient sulfur transfer reagent. Nucleosides Nucleotides Nucleic Acids. 22:461-468.

52. Drygin, D., Barone, S, and Bennett, C.F. 2004 Sequence-dependent cytotoxicity of secondgeneration oligonucleotides. Nucleic Acids Res. 32:6585-6594.

53. Drygin, D., Koo, S., Perera, R., Barone, S., and Bennett, C.F. 2005. Induction of toll-like receptors and NALP/PAN/PYPAF family members by modified oligonucleotides in lung epithelial carcinoma cells. Oligonucleotides. 15:105-118.

54. Winer, J., Jung, C.K., Shackel, I., and Williams, P.M. 1999. Development and validation of real-time quantitative reverse transcriptase-polymerase chain reaction for monitoring gene expression in cardiac myocytes in vitro. Anal. Biochem. 270:41-49.

55. Leeds, J.M., Graham, M.J., Truong, L., and Cummins, L.L. 1996. Quantitation of phosphorothioate oligonucleotides in human plasma. Anal. Biochem. 235:36-43.

56. Geary, R.S., et al. 1997. Antisense oligonucleotide inhibitors for the treatment of cancer: 1 . Pharmacokinetic properties of phosphorothioate oligodeoxynucleotides. Anticancer Drug Des. 12:383-393. 